Sección uno: Ensayo

Radiografía de la innovación educativa en el Siglo XXI

\title{
El uso de videoclips en la enseñanza de la destreza lectora en lengua extranjera con adultos universitarios ${ }^{1}$
}

The use of videoclips to teach foreign language reading skills to university students

Carmen María Toscano-Fuentes

Universidad de Huelva

carmen.toscano@dfing.uhu.es

Analí Fernández-Corbacho

Universidad de Huelva

anali.fernandez@dfing.uhu.es

\section{Resumen}

En niveles universitarios se espera que las destrezas lectoras de los aprendices estén totalmente desarrolladas, ya que son claves en el éxito académico y profesional. Sin embargo, existen evidencias de que un número significativo de pregraduados universitarios con clases de lengua extranjera presentan dificultades en la fluidez lectora silenciosa, lo que dificulta su comprensión lectora y la adquisición de conocimientos. Debido a la estrecha relación entre la aptitud musical y la destreza lectora, la utilización de videoclips musicales en el aula de lengua extranjera puede tener un impacto positivo para el aprendizaje. Tras medir la fluidez lectora silenciosa de 46 estudiantes universitarios y comprobar la relación entre esta y su aptitud musical, se puso en marcha una experiencia educativa. Para ello se analizó el tipo de videoclips que el alumnado consumía en LE y se diseñó una experiencia didáctica con videoclips y herramientas TACs. Se comprobó la acogida de dicha innovación docente en el aula, y los posibles beneficios para mejorar el aprendizaje. Los resultados y comentarios recogidos muestran el potencial del uso de videoclips subtitulados y de la creación de kahoots con preguntas de comprensión relativas a los videoclips para mejorar conocimiento de la LE.

Palabras claves: fluidez lectora silenciosa; aptitud musical; videoclips subtitulados; experiencia educativa

${ }^{1}$ Recibido: 10/01/2020 Evaluado: 08/02/2020 Aceptado: 09/02/2020 


\begin{abstract}
At university levels, the reading abilities of the students are expected to be fully developed, as they are key to academic and professional success. However, there is evidence that a significant number of university undergraduates with foreign language classes present difficulties in silent reading fluency, which hinders their reading comprehension and knowledge acquisition. Due to the close relationship between musical aptitude and reading skills, the use of videoclips in the foreign language classroom can have a positive impact on learning. After measuring the silent reading fluency of 46 university students and checking the relationship with their musical aptitude, an educational experience was launched. For this, the type of videoclips that students usually consume in the FL was analyzed and a didactic experience with videoclips and LKT was designed. The teaching innovation in the classroom, and the possible benefits to improve learning were verified. The results and comments collected show the benefits of using subtitled videoclips and creating kahoots with comprehension questions about the videoclips improve the knowledge of FL.
\end{abstract}

Key words: silent reading fluency, musical aptitude, subtitled videoclips, learning experience

\title{
Introducción
}

Una destreza lectora plenamente desarrollada es un instrumento esencial de acceso a la información que está íntimamente relacionado con el éxito académico, social y profesional. Si no comprendemos lo que leemos, no podemos acceder al conocimiento, mermándose así nuestra capacidad de aprendizaje. Los niveles educativos primarios y secundarios ponen especial énfasis en el desarrollo de la lectura tanto en lengua materna como en la lengua extranjera. Sin embargo, pruebas a nivel nacional e internacional (PISA, PIRLS, ESLC) sitúan a España y en especial a Andalucía por debajo de la media nacional y europea, a pesar del esfuerzo de los centros educativos por mejorarla con planes de lectura. En el nivel universitario también se han detectado problemas lectores en los estudiantes de lenguas extranjeras en los primeros cursos de grado (Foncubierta, Herrero y Fonseca-Mora, 2018).

En el proceso lector intervienen numerosas variables relacionadas con las diferencias y semejanzas entre la lengua materna (L1) y la lengua extranjera (LE); siendo las de índole ortográfica y fonológica sumamente importantes, sobre todo en los niveles iniciales; pues permiten establecer la relación entre la forma oral y escrita de las palabras, y de ese modo, reconocer el vocabulario oral que posee el lector novel (Sparks, Patton, Ganschow, Humbach, y Javorsky, 2008). Sin embargo, otros factores lingüísticos también influyen en el desarrollo lector. Así, como mantiene Scarborough (2002), aunque un niño pueda decodificar perfectamente un texto, no lo comprenderá (1) si no conoce la palabra en su forma oral, (2) si no comprende las relaciones sintácticas y semánticas entre las palabras, o (3) si carece de conocimiento base o de estrategias de interpretación del texto. No obstante, son muchos más los factores, lingüísticos, cognitivos y sociales que tienen un impacto en el desarrollo lector en LE (Fernández- Corbacho, 2018). 
Con el fin de facilitar el estudio y los enfoques pedagógicos de la destreza lectora, ésta se divide en cinco componentes: conciencia fonológica, principio alfabético, fluidez, vocabulario y comprensión (NICHD, 2000); considerándose la conciencia fonológica la piedra angular sobre la que se sustenta la adquisición de la destreza lectora (Goswami, 2009). Para comprender lo que se lee es imprescindible que el lector sonorice el texto (FonsecaMora, 2013) y sea capaz de conectar las grafías con los sonidos y poder así decodificarlo y entenderlo. Tener la capacidad de identificar sonidos permite asociar los fonemas con su representación escrita (Bradley y Bryant, 1983; Goswami, 1990; Moreno et al., 2009); por lo que el aprendiz que tiene desarrollada la capacidad auditiva para distinguir y manipular fonemas cuenta con una ventaja en el momento de conectar los elementos fonológicos y ortográficos (Anvari et al., 2002). A su vez, el reconocimiento automático de las palabras permite leer con fluidez. Esto implica hacerlo con rapidez, expresividad y corrección, paso previo necesario para alcanzar la comprensión lectora. En la edad adulta, la lectura se suele realizar de forma silenciosa. En numerosas ocasiones el estudiante carece de un buen nivel de fluidez lectora silenciosa, que actúa como elemento mediador entre las habilidades de codificación y comprensión. Los dos elementos clave en el modelo conocido como Visión Simple de la Lectura (Gough y Tunmer, 1986; Hoover y Gough, 1990). Por otra parte, cuando se trata de la lectura en una LE, los tres pilares del desarrollo lector son la destreza lectora en L1, las habilidades de decodificación en la L2 y el conocimiento de la L2 (Goswami, 2005). Es más, en el estudio de la relación entre la fluidez lectora y la comprensión lectora en LE, el conocimiento gramatical de la lengua meta parece ser clave (Jeon y Yamashita, 2014).

Numerosos estudios afirman que una buena capacidad musical favorece el proceso lector, ya que música y lenguaje comparten características comunes tales como la discriminación melódica, rítmica y armónica, el reconocimiento de los sonidos, entre otros (Bolduc, 2008; Standley, 2008; Lessard y Hall, 2011; Toscano- Fuentes, 2010; Toscano-Fuentes y FonsecaMora, 2012). Por otro lado, hay un buen número de estudios que aseguran que estudiantes con una alta capacidad musical presentan menos dificultades en la lectura y que el entrenamiento musical incide directamente en el proceso lector específicamente en las habilidades fonológicas (Chobert y Besson, 2013; Tierney, Krizman y Kraus, 2015). Más específicamente, las canciones fusionan tanto la información lingüística como la musical, involucrando a ambas partes del hemisferio a través del cuerpo calloso, el cual se fortalece al transmitir los mensajes entre los hemisferios” (Toscano-Fuentes y Fonseca-Mora, 2012, p.203), estrecha la relación entre fonema-grafema y ayuda a aprender nuevo vocabulario y estructuras gramaticales. Así mismo, promueve la retención de palabras y sonidos en la memoria de trabajo, especialmente cuando se origina el bucle fonológico, con lo que mejora la fluidez y la eficacia de los procesos lectores. Bastante se ha investigado en educación primaria y secundaria sobre la mejora de la destreza lectora a través de entrenamientos musicales (Barwick, Valentine, West, y Wilding, 1989; Kim, Petscher y Foorman, 2015). Sin embargo, en una población adulta, los estudios son menos numerosos (Amitay, Ahissar y Nelken, 2002; Foxton, Talcott, Witton, Brace, McIntyre, y Griffiths, 2003). Como Tucker (1981, p.16) afirma "the use of music with older students, even college students, as a tool for enhancing reading ability, is not as well documented, mainly because of the scarcity of research fusing music and reading beyond the primary grades”. 
Por otra parte, los videoclips son un recurso aún mejor que las canciones porque fomentan el aprendizaje de las TICs y las TACs (Tecnología del Aprendizaje y el Conocimiento), además de promover el desarrollo de la competencia digital propuesta por las leyes educativas actuales. También ofrecen imágenes y subtítulos que pueden beneficiar el proceso lector en el alumnado de lengua extranjera. Estudios de Educación Primaria (Toscano-Fuentes y Julián de Vega, 2018; Gertrudix-Barrio y Rivas-Rebaque, 2015), Educación Secundaria (GertrudixBarrio y Gertrudix-Barrio, 2010; Berk, 2008) y Universidad (Mericka, 2018 y SánchezVizcaino y Fonseca-Mora, 2018) aconsejan la incorporación de este recurso debido al aumento del input auditivo, de la motivación, de la mejora de la conciencia fonológica y de la fluidez lectora así como de la competencia audiovisual y emocional.

Una de las plataformas más recurrentes para la búsqueda de videoclips es Youtube. CancelaOuviña y Herrera-Barba (2014) aseguran que, además de ser un recurso actual que promueve las TICS y ayuda a mejorar el aprendizaje de una lengua extranjera, fomenta el aprendizaje autónomo del alumnado, mejorando así no solo la competencia digital sino el aprender a aprender. Por tanto, el rol del alumno ya no es un mero reproductor de contenidos memorísticos sino que “debe llegar a ser un usuario inteligente y crítico de la información, para lo que precisa aprender a buscar, obtener, procesar y comunicar información y convertirla en conocimiento” (Segura, Candioti, y Medina, 2007, p. 6).

El presente trabajo pretende, por un lado, analizar la relación entre la fluidez lectora silenciosa y la aptitud musical del alumnado universitario de pregrado, y, por otro lado, llevar una experiencia educativa al aula de primaria en el que, primeramente, se recopila un listado de los videoclips musicales más consumidos por los estudiantes universitarios y se elabora una serie de kahoots con preguntas de comprensión relativas a esos videoclips con el fin de mejorar tanto el conocimiento de la LE como las destrezas lectoras.

\section{Objetivos e hipótesis}

Los objetivos que se plantean en el presente estudio son los siguientes:

1. Identificar el nivel de fluidez lectora silenciosa en lengua materna y en lengua extranjera (inglés) de alumnado universitario de pregrado.

2. Estudiar la relación entre la fluidez lectora silenciosa y el nivel en la lengua extranjera.

3. Determinar la aptitud musical de los participantes con el fin de observar si existe una relación entre su aptitud musical y la fluidez lectora silenciosa.

4. Registrar los videoclips más consumidos por los estudiantes universitarios participantes en el estudio y hacerles reflexionar sobre las temáticas y los mensajes de dichos videoclips musicales y su idoneidad para el aprendizaje de la lengua extranjera.

5. Poner en marcha una experiencia educativa basada en videoclips musicales dentro del programa de formación de futuros maestros de primaria y evaluar dicha experiencia. 


\section{Método}

\section{Participantes}

En este estudio han participado 46 estudiantes universitarios de pregrado, de edades comprendidas entre los 18 y 50 años. Todos ellos cursan carreras no lingüísticas, aunque tienen alguna asignatura en lengua extranjera. Para la parte del proyecto en la que se pone en marcha una experiencia educativa basada en el uso de videoclips, se seleccionan 23 participantes, pertenecientes a un grupo que está formado por futuros maestros de Educación Primaria.

\section{Instrumentos y Materiales}

Los instrumentos y materiales usados se presentan siguiendo las fases en las que se dividió el estudio.

Fase 1: Estudio de la fluidez lectora silenciosa, la comprensión lectora y la aptitud musical.

En esta primera parte del estudio se emplearon tres instrumentos para la recogida de datos cuantitativos.

- Dialang. Es un test diseñado por la Universidad de Lancaster, online y de libre acceso, que permite el diagnóstico del nivel en la lengua extranjera. En este estudio, se seleccionó la prueba de comprensión lectora en inglés.

- Test de fluidez lectora silenciosa en inglés y español. Se trata de un test de elaboración propia, basado en otros tests similares, que mide la rapidez con la que los estudiantes separan las palabras de un texto. Estas aparecen sin espacios ni signos de puntuación. Es un test cronometrado, la puntuación final es el total de palabras correctamente separadas (identificadas) en 3 minutos.

- Test de aptitud musical MiniPROMPS, desarrollado por la Universidad de Innsbruck. Es un test online, de acceso libre, que permite medir varios elementos de la aptitud musical: melodía, ritmo, tono y acento. Al finalizar el test, se obtiene una puntuación global.

Fase 2: Recopilación de los videoclips más consumidos por los estudiantes.

- Google forms se han utilizado para recopilar la selección de videoclips llevada a cabo por los estudiantes. Esta herramienta permite el cálculo de datos y crea gráficos a partir de los mismos.

- Observación directa del aula por parte de la docente. Se realizaron actividades de reflexión y debate durante el análisis de algunos de los videoclips seleccionados. Las docentes tomaron nota de los comentarios de los estudiantes. 
- Preguntas de reflexión sobre el uso de videoclips para el aprendizaje de una LE. Los estudiantes anotaron de forma anónima algunas reflexiones a partir de preguntas realizadas por las docentes.

Fase 3: Puesta en marcha de una experiencia educativa basada en el uso de videoclips.

- TACs: La aplicación de las nuevas tecnologías en el aprendizaje y en la comunicación a través de dos aplicaciones: Kahoot, para hacer tests interactivos online, y Subtitle Workshop, para poner subtítulos a los vídeos que carecían de ellos.

- Observación directa del aula por parte de la docente.

- Preguntas de reflexión en el aula para obtener retroalimentación de los estudiantes sobre la experiencia educativa.

\section{Procedimiento}

La investigación se ha dividido en tres periodos bien diferenciados. En primer lugar, se recogieron datos cuantitativos y se analizaron los resultados. Seguidamente, se llevó a cabo la recopilación de videoclips y análisis de los mismos. Finalmente se puso en marcha la experiencia educativa. A continuación, se describen detalladamente cada una de las fases.

Fase primera:

Durante el primer trimestre del curso académico se realizó la recopilación y adaptación de las pruebas necesarias y la administración de las mismas. Los participantes comenzaron con Dialang de forma individual en aulas de informática. En la primera parte, los estudiantes obtuvieron una puntuación en base a las palabras que reconocían como reales en la LE. Según el nivel alcanzado, el programa proponía una serie de actividades de comprensión lectora. Finalmente se obtuvo un resultado expresado en números y en niveles según el MCERL. La prueba total duró aproximadamente 30-40 minutos, dependiendo del nivel de cada estudiante. Seguidamente, se administró la prueba de aptitud musical MiniPROMPS. Esta también se realizó de forma individual en el aula de informática; en este caso cada estudiante disponía de auriculares para facilitar la realización de la misma. Esta prueba duró entre 20-30 minutos. Ambos tests se hicieron durante una misma sesión lectiva.

Respecto a la prueba de fluidez lectora silenciosa, se realizó primero en la LE y unos días más tarde en la L1. Es una prueba que se administró de forma grupal pues consistía en la segmentación de palabras de un texto que carecía de espacios y signos de puntuación. Se trataba de una prueba cronometrada y permitía medir la velocidad y corrección con la que los estudiantes identificaban visualmente las palabras en un texto. Los textos seleccionados en ambas lenguas pertenecían a diferentes apartados de la Declaración de Derechos Humanos. 
Fase segunda:

Tras la recogida y análisis de los datos los estudiantes seleccionaron los 10 videoclips que más le gustaban o que más escuchaban en lengua extranjera. Para ello completaron un cuestionario online de Googleforms en el cual tenían que indicar, por orden de preferencia, los 10 videoclips en lengua inglesa que más consumían habitualmente, se les pidió además que indicaran el artista o grupo, el nombre de la canción y el enlace de YouTube para ver el videoclip. De ese modo se evitaron malentendidos con los nombres o autores de las canciones y se accedía directamente al videoclip, pues generalmente existen varias versiones para una misma canción. Posteriormente, se presentaron los resultados en el aula, y se discutió sobre los más populares. Esta parte de la experiencia tuvo un enfoque marcadamente reflexivo, pues como apunta el modelo de aprendizaje experiencial (Kolb y Kolb, 2008), la reflexión es un elemento clave para que haya aprendizaje. Así, durante el análisis de los videoclips se pidió a los estudiantes que prestaran atención a los elementos culturales y los mensajes que contenían. Seguidamente, se les llevó por un proceso de reflexión, no solo sobre las aportaciones culturales de los vídeos, sino también sobre si los consideraban adecuados o atractivos para el uso didáctico en el aula, si pensaban que les podían ayudar en el aprendizaje de la LE y de qué forma. También se les preguntó si ya utilizaban o habían utilizado ellos mismos videoclips musicales para aprender una LE.

Fase tercera:

En esta fase final se seleccionó a un grupo de 23 estudiantes, futuros maestros de Educación Primaria, que estaban matriculados en una asignatura de metodología de enseñanza del inglés como lengua extranjera. El objetivo principal de la experiencia didáctica era promover el uso de videoclips como herramientas de aprendizaje y enseñanza de lenguas extranjeras, de modo que transfirieran lo vivido hasta el momento y reflexionaran sobre la posibilidad de utilizar videoclips en el aula de primaria. Para ello (1) tuvieron que seleccionar videoclips apropiados para la edad y los contenidos didácticos, (2) añadir subtítulos a los vídeos que no los tuvieran; y (3) preparar preguntas de comprensión basadas en los videoclips, utilizando Kahoot.

\section{Resultados y discusión}

Objetivo 1: Identificar el nivel de fluidez lectora silenciosa en lengua materna y en lengua extranjera (inglés) de alumnado universitario de pregrado.

Para medir la fluidez lectora silenciosa de los estudiantes universitarios que participaron en este estudio se computaron únicamente las palabras que habían sido correctamente separadas en los textos. Como se podía prever la media de palabras separadas correctamente por minuto es mayor en español $(114,259 \mathrm{p} / \mathrm{m})$ que en inglés LE $(67,996 \mathrm{p} / \mathrm{m})$. Los estudiantes universitarios son capaces de leer prácticamente el doble de rápido en la L1 que en la LE. Sin embargo, llama la atención que incluso el nivel de fluidez lectora silenciosa en la LE está bastante por debajo de la norma, 280 palabras por minuto (Hasbrouck y Tindal, 2006). Hecho que coincide con lo que apunta el estudio de Foncubierta et al. (2018). 
Si comparamos los niveles de fluidez lectora silenciosa en la L1 y la LE, los análisis estadísticos realizados indican que existe una correlación leve entre la L1 y la LE $(\rho=.253$, $\mathrm{p}=$.017). Esto parece señalar que los lectores españoles que son capaces de reconocer más palabras en español, por líneas generales, también son capaces de reconocerlas en inglés. La transferencia de la habilidad decodificadora, que permite el reconocimiento de palabras, de la L1 a la L2 es un hecho constatado en edades más tempranas (Fonseca-Mora y FernándezCorbacho, 2017; Ziegler y Goswami, 2006); sin embargo, al igual que el estudio de Foncubierta et al. (2018), nuestros datos apuntan a que los efectos de dicha transferencia parecen seguir presentes en la población universitaria. Parece, por tanto, que la experiencia lectora de los estudiantes universitarios en la L1 les permite transferir a la LE su habilidad para decodificar palabras visualmente.

Objetivo 2: Estudiar la relación entre la fluidez lectora silenciosa y el nivel en la lengua extranjera.

Por otra parte, se quiso averiguar si el nivel de fluidez lectora silenciosa tenía relación con la comprensión lectora. En la Tabla 1 se puede observar que al agrupar a los estudiantes según el nivel de comprensión lectora en LE obtenido en la prueba de Dialang, aparecen diferencias entre los grupos con respecto a la cantidad de palabras que son capaces de decodificar visualmente en el texto.

Tabla 1. Comparación de resultados en fluidez lectora silenciosa en L1 y LE según nivel del MCER

\begin{tabular}{ccc}
\hline Nivel & Fluidez lectora LE & Fluidez lectora L1 \\
B2 $-\mathbf{C 1}$ & $80 \mathrm{p} / \mathrm{m}$ & $118,6666667 \mathrm{p} / \mathrm{m}$ \\
A2-B1 & $69,82352941 \mathrm{p} / \mathrm{m}$ & $123,1176471 \mathrm{p} / \mathrm{m}$ \\
A1 & $54,16666667 \mathrm{p} / \mathrm{m}$ & $101 \mathrm{p} / \mathrm{m}$ \\
\hline $\mathrm{p} / \mathrm{m}=$ palabras por minuto. &
\end{tabular}

Sin embargo, los datos en español no son significativos. De hecho, aunque se observa que los estudiantes que obtuvieron un nivel A1 presentan una menor fluidez lectora, la media del grupo A2-B1 es superior al grupo B2-C1. Por tanto, el nivel de fluidez lectora en la L1 no tiene relación con la comprensión en la LE.

En cuanto a la LE, sí se observa que a mayor nivel obtenido en las pruebas de comprensión lectora Dialang, mayor fluidez lectora en LE. Como apuntaban Jeon y Yamashita (2014), es necesario el conocimiento de la LE para poder comprender lo que se lee. Puesto que la prueba Dialang también incluye una prueba de discriminación de palabras reales y no reales en la LE, en cierto modo se mide el conocimiento de la LE; con lo que podría ser una prueba válida para constatar la existencia de una relación entre la fluidez en comprensión lectora en LE. No obstante, sería necesario ampliar la muestra para obtener resultados concluyentes. 
Objetivo 3: Determinar la aptitud musical de los participantes con el fin de observar si existe una relación entre su aptitud musical y la fluidez lectora silenciosa.

Seguidamente, se compararon los resultados obtenidos en destrezas lectoras en L1 y LE y las puntuaciones obtenidas en el test de aptitud musical. Como se observa en la Tabla 2, en general, los estudiantes con mayores niveles de comprensión y fluidez lectora, también tienen una mayor aptitud musical.

Tabla 2. Comparación de resultados en aptitud musical y fluidez lectora según niveles del MCER

\begin{tabular}{cccc}
\hline Nivel & MiniPROMS & Fluidez lectora LE & Fluidez lectora L1 \\
B2 - C1 & 20,875 & $80 \mathrm{p} / \mathrm{m}$ & $118,6666667 \mathrm{p} / \mathrm{m}$ \\
A2-B1 & 17 & $69,82352941 \mathrm{p} / \mathrm{m}$ & $123,1176471 \mathrm{p} / \mathrm{m}$ \\
A1 & 13,15 & $54,16666667 \mathrm{p} / \mathrm{m}$ & $101 \mathrm{p} / \mathrm{m}$ \\
\hline * $/ \mathrm{m}$ = palabras por minuto & &
\end{tabular}

${ }^{*} \mathrm{p} / \mathrm{m}=$ palabras por minuto.

Curiosamente, esta tendencia, está más marcada en relación a la LE. En consonancia con otros estudios recientes, nuestros resultados apuntan a que existe una relación entre la destreza lectora y la aptitud musical. Esto respaldaría la conveniencia de promover experiencias didácticas en las aulas de LE que incluyan de forma significativa el uso de la música. Como se ha mencionado anteriormente, si además del input oral, el aprendiz estuviera expuesto a la letra de las canciones a la vez, se mejoraría el aprendizaje de la LE.

Objetivo 4: Registrar los videoclips más consumidos por los estudiantes universitarios participantes en el estudio y hacerles reflexionar sobre las temáticas y los mensajes de los videoclips musicales y su idoneidad para el aprendizaje de la lengua extranjera.

En la segunda fase del estudio los estudiantes completaron un cuestionario online, indicando, por orden de preferencia, los 10 videoclips musicales en lengua inglesa que más veían. También especificaron el artista o grupo, el nombre de la canción y el enlace de YouTube para ver el videoclip. La Tabla 3 presenta los 10 videoclips más votados.

Tabla 3. Videoclips más seleccionados en inglés (por orden de preferencia)

Título de la canción $\quad$ Nombre de artista/grupo $\quad \mathbf{N}^{\circ}$ de veces elegido $\quad$ Enlace

$\begin{array}{cccc}\begin{array}{c}\text { Shape of you } \\ \text { All of me }\end{array} & \text { Ed Sheeran } & \text { 23 Elecciones } & \text { https://bit.ly/2S8HeZ4 } \\ \underline{\text { Perfect }} & \text { John Legend } & \text { 21 Elecciones } & \text { https://bit.ly/2yIev6N } \\ \underline{\text { Halo }} & \text { Ed Sheeran } & 16 \text { Elecciones } & \text { https://bit.ly/3bE2Xjs } \\ & \text { Beyonce } & 13 \text { Elecciones } & \text { https://bit.ly/2xXBTwY }\end{array}$

No 8, 2020. Página | 53 


\begin{tabular}{cccc}
\hline Título de la canción & Nombre de artista/grupo & $\mathbf{N}^{\mathbf{0}}$ de veces elegido & Enlace \\
\hline$\underline{\text { Photograph }}$ & Ed Sheeran & 12 Elecciones & https://bit.ly/2KvUzq7 \\
$\underline{\text { Diamonds }}$ & Rihanna & 12 Elecciones & https://bit.ly/358LlKe \\
$\underline{\text { Havana }}$ & Camila Cabello & 11 Elecciones & https://bit.ly/2VXTgpl \\
$\underline{\text { Let her go }}$ & Passenger & 11 Elecciones & https://bit.ly/2xP6NI1 \\
$\underline{\text { Someone like you }}$ & Adele & 11 Elecciones & https://bit.ly/2yEClQT \\
Imagine & John Lennon & 9 Elecciones & https://bit.ly/3aFn3bE \\
\hline
\end{tabular}

A partir de la selección de los videoclips se inició el proceso de reflexión sobre los valores culturales que se transmitían. Se trabajó utilizando dinámicas de grupo cooperativos para negociar la información. En primera instancia, se invitó al alumnado participante primero a determinar qué tipo de valores aparecían reflejados en los vídeos. Se dieron cuenta de que una gran mayoría (80\%) hacen referencia a las relaciones interpersonales, generalmente, amorosas (por ejemplo, Someone like you de Adele, Let her go de Passenger, Photograph y Perfect, ambas de Ed Sheeran, All of Me de John Legend, y Diamonds de Rihanna). Otros videos, servían para promocionar un destino turístico y un estilo de vida (Havana de Camila Cabello), o reflejaban valores que promovían la paz (Imagine de John Lennon). Sin embargo, las canciones tendían a ofrecer una visión idealizada de las relaciones y una estudiante subrayó la importancia de ser conscientes de que son canciones, no la vida real, algo que otros reconocían olvidar. Posteriormente, se les preguntó si, como ciudadanos europeos, veían valores o mensajes relacionados con la cultura europea. Tuvieron problemas para llegar a un acuerdo sobre qué es la cultura europea, de hecho, no fueron capaces de definirla y concluyeron que la cultura nacional prevalece. De todas formas, entre los vídeos seleccionados no reconocieron elementos identificativos de Europa, sino más bien ejemplos de una cultura globalizada que todos aceptamos como propia (comportamientos, formas de vestir, relaciones interpersonales, etc.). Como valoración global, llegaron a la conclusión de que la gran mayoría de los videoclips que consumían no reflejaban valores europeos específicamente, sino que eran videos y canciones internacionales, principalmente estadounidenses y británicos; música comercial y de actualidad, creada para bailar y disfrutar no para provocar reflexiones. Reconocieron que ellos no hubieran llegado a reflexionar al respecto de no ser por la actividad que estaban realizando en clase.

Finalmente, se debatió con el alumnado sobre el potencial del uso de los videoclips seleccionados por ellos mismos para aprender una lengua extranjera, así como sobre su uso como material didáctico para enseñar en el aula. De primera reflexión señalaron que, aunque no era el caso de los vídeos que habían seleccionado, muchos videoclips reflejan valores sexistas, machistas, incluso violentos, y por lo tanto no son aptos para su uso como material de enseñanza y aprendizaje. Después, se les hizo una serie de preguntas para que escribieran 
algunas reflexiones. De forma recurrente aparecieron varios temas en sus repuestas. La introducción de videoclips en el aula de LE se considera un elemento innovador, que hace el aprendizaje más ameno y dinámico pues se aprende "sin darse cuenta”. Respecto a los beneficios que percibían usando videoclips subtitulados, los más mencionados fueron: mejora de la pronunciación, comprensión oral, fluidez lectora ("nos sirve para leer más rápido”), aprendizaje de gramática y vocabulario, facilita la comprensión de conceptos porque ayudan a "darle sentido a las palabras”, "dan más sentido que frases de vocabulario”, y en general sienten que mejora su nivel de inglés. Algunos mencionaron incluso la formación de oraciones y la escritura, así como la calidad de sus producciones orales, pues le ayudan a que "lo que diga tenga concordancia". También afirman que el uso de videoclips mejora la atención, poder recordar "todo mejor", por ser "divertido y pegadizo"; las canciones mejoran su motivación: "pues con ellas expresamos nuestros sentimientos, forman parte de nuestra vida”, “te llena de energía y vitalidad”, se aprende más “al ser algo que nos interesa”, pues están “interiorizado el idioma con la música”. Un 30\% de los estudiantes ya utilizaban música para aprender inglés, uno de ellos sí admitió escuchar música, pero no había sido consciente hasta ahora de todo lo que había aprendido. En el caso de otro estudiante, la experiencia en el aula le ha hecho consciente de que "sí que soy capaz de aprender significados de palabras viendo videoclips en inglés subtitulados”. Se aprecia en sus comentarios una enorme sensación de autoeficacia y éxito; y expresan su deseo de seguir aprendiendo de este modo y que más docentes de lenguas extranjeras los utilicen en clase.

Objetivo 5: Poner en marcha una experiencia educativa basada en videoclips musicales dentro del programa de formación de futuros maestros de primaria y evaluar dicha experiencia.

Para el grupo de 23 estudiantes del Grado en Educación Primaria, se realizaron actividades específicas para ayudarles a transferir los conocimientos y experiencias acumulados hasta el momento, reflexionar sobre el uso de videoclips en el aula de primaria y diseñar ellos mismos actividades basadas en un videoclip. Se les indicó que el objetivo de las actividades tenía que ir más allá de escuchar una canción o ver un videoclip, pues se trataba de trabajar las destrezas lectoras (decodificación, fluidez y comprensión). Por tanto, era necesario que los videoclips estuvieran subtitulados, y tenían que trabajar con ellos la comprensión lectora. El hecho de convertirse ellos mismos en diseñadores de materiales y tener la posibilidad de crear una batería de videoclips con ejercicios de destreza lectora para poder usar en sus aulas, fue algo que les motivó desde el principio.

Durante la selección de videoclips para alumnado de primaria, surgió un tema de debate y es que la selección de videoclips que ellos consumían y que estaba en auge en ese momento no era adecuado para su uso en Educación Primaria, bien porque el nivel de inglés era más elevado del que el estudiante presentaba, bien porque el contenido no era el adecuado o porque las imágenes que se mostraban eran inapropiadas para la práctica docente. Debido a esto, se les dejó libertad para que ellos decidieran si seleccionaban un videoclip actual para poder trabajar su propia competencia en lengua extranjera o elegían uno para uso didáctico en primaria.

La mayoría de los estudiantes (20/23) seleccionaron un videoclip adecuado para su uso didáctico en el aula y así poder contar con una batería de kahoots para incorporar en el aula 
de lengua extranjera cuando comenzaran a trabajar en el campo de la enseñanza. Casi todas las canciones provenían de canales específicos de enseñanza de inglés como lengua extranjera, por ejemplo, Super Simple Songs; Singing Walrus- English Songs for Kids; Maple Leaf Learning; o Dream English Kids.

Tras seleccionar el videoclip, si este no estaba subtitulado, le añadieron subtítulos, ellos mismos buscaron las aplicaciones y decidieron usar el programa Subtitle Workshop. Por último, crearon un kahoot con preguntas basadas en el texto de la canción. Para esta última parte, al comienzo de la sesión se les explicó el funcionamiento de Kahoot, así como los diferentes formatos que la plataforma presentaba, de manera que pudieran elegir el tipo de preguntas que querían hacer. Los formatos que ofrece la aplicación son los siguientes:

1. Quiz: A cada jugador se le da una pregunta con cuatro respuestas de las cuales solamente una es la correcta.

2. True o false: A cada jugador se le da una pregunta con una respuesta que debe marcar como verdadera o falsa.

3. Open-ended: Cada jugador debe dar una respuesta abierta a la pregunta.

4. Puzzle: Cada jugador tiene que ordenar las respuestas correctamente.

Debido a las edades para las que estaban destinadas las actividades, se seleccionó principalmente los formatos quiz, y true/false.

Las imágenes 1 y 2 muestran ejemplos de kahoots diseñados por los participantes.

\section{What does he have to put on?}

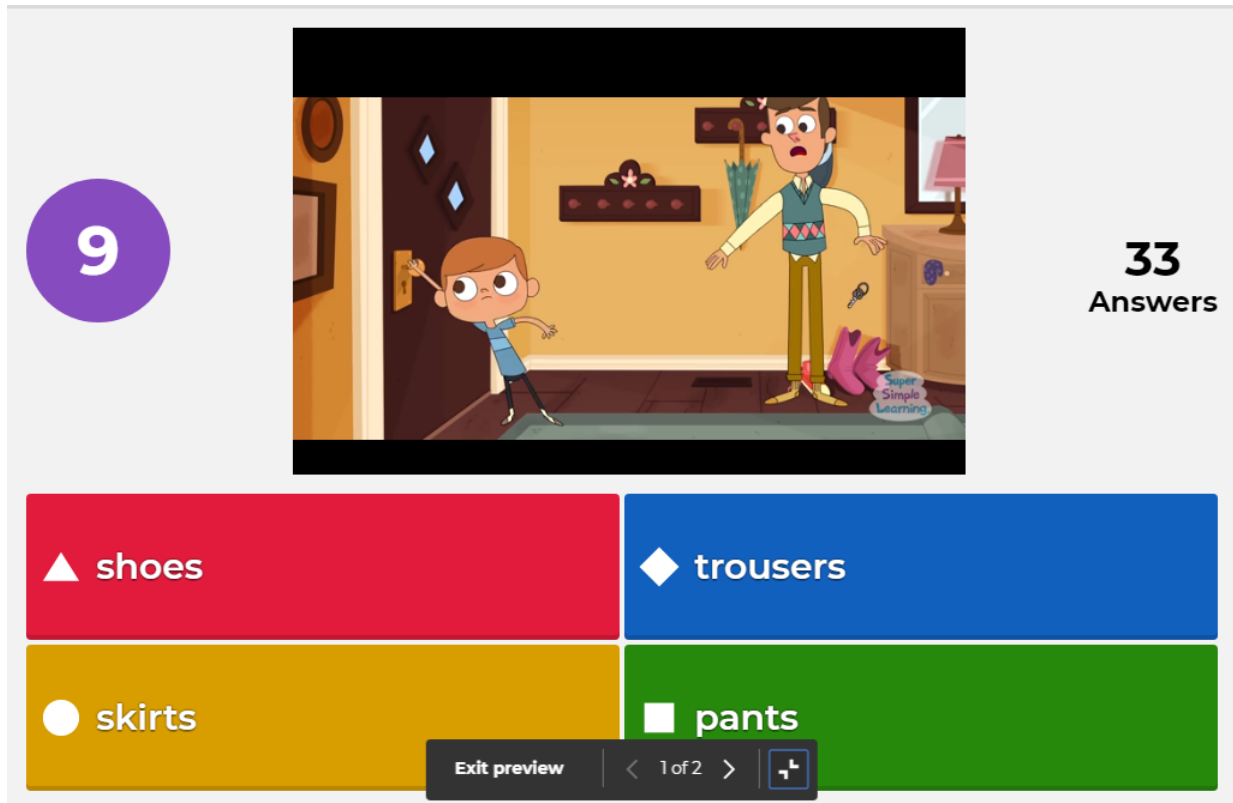

Imagen 1.Pregunta de comprensión auditiva y decodificación de palabras en Kahoot. 


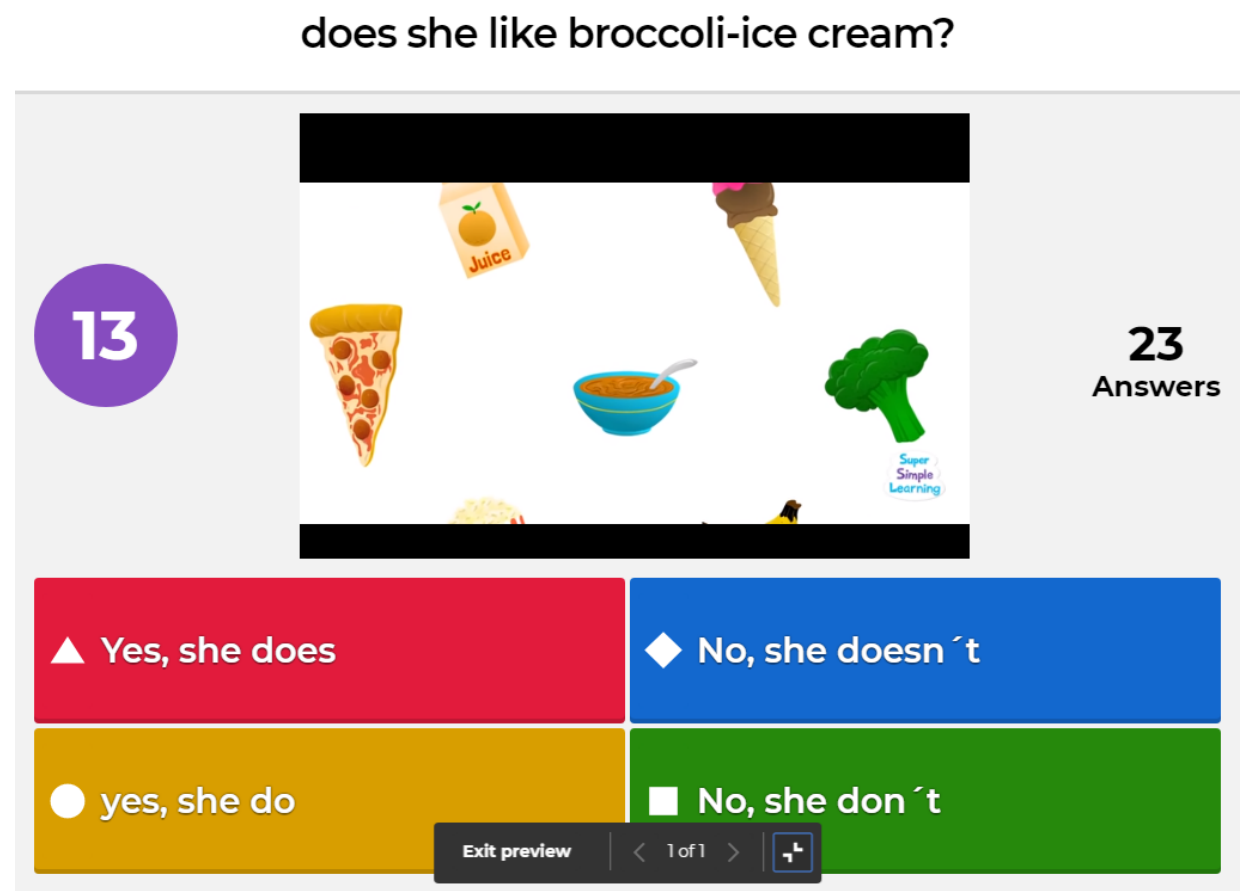

Imagen 2. Pregunta de comprensión auditiva y corrección gramatical en Kahoot.

Durante todo el proceso se incentivó el uso no solo de las TICs sino de las TACs, es decir, un uso pedagógico de las tecnologías, lo cual potencia el aprendizaje (Velasco Rodríguez, 2017).

Finalmente, se debatió con el alumnado sobre la experiencia educativa. Los participantes aseguraron que la experiencia había sido muy enriquecedora y señalaron varios motivos:

- Aprendieron a utilizar una plataforma gratuita la cual permitía la creación de cuestionarios para la comprensión auditiva-lectora del videoclip.

- Consideraron la herramienta como atractiva y motivante para el estudiante en contraposición al tradicional worksheet.

- Les sirvió para reflexionar sobre la importancia de seleccionar adecuadamente canciones, temática y nivel educativo al que van dirigidas las actividades que se diseñan, pues no todas las canciones, por muy actuales y populares que sean, son adecuadas desde el punto de vista didáctico.

- Consiguieron una batería de videoclips con preguntas de comprensión, útiles para su futura práctica docente.

Por otro lado, observaron que este tipo de recursos hay que adaptarlos a la realidad de cada centro, puesto que para su utilización se necesita un móvil o tablet por estudiante. Sin embargo, ellos mismo ofrecieron soluciones para esta situación, por ejemplo, se les podía dar un folio con todas las respuestas para que de manera individualizada seleccionarán en el papel la respuesta correcta y el profesorado la corregía posteriormente; o se les daba una cartulina de cada uno de los colores de la respuesta para que mostraran la adecuada; o trabajaban en grupo y si el colegio disponía de ordenadores cada grupo podía responder las 
preguntas planteadas por el profesorado de manera que sus respuestas se pudieran ver en la pantalla. Todos coincidieron en que, si se utiliza el papel con esta aplicación, se pierde la filosofía del juego pues desaparece la emoción de ver quién acaba el primero y comparar los puntos que el programa otorga por acertar lo más rápido posible. Pero si no existe la posibilidad de utilizar ordenador o móviles, hay que ofrecer alternativas adecuadas para ser empleadas en todos los centros y contextos educativos.

Esta experiencia didáctica no solo fue aplaudida por los futuros maestros de primaria que participaron en este estudio, ya que tuvieron la posibilidad de poner en práctica en un aula real los videoclips y kahoots que habían diseñado (Toscano-Fuentes y Julián de Vega, 2018). De modo que vivieron en primera persona el entusiasmo con que el alumnado de primaria acogió la actividad, pues les encanta todo lo que implique juego competitivo, ya que mientras juegan aprenden sin ser conscientes de ello. Gracias a este enfoque experiencial el alumnado de nuestro estudio pudo comprobar por sí mismos la eficacia, idoneidad, así como las posibilidades de mejora de los materiales que habían diseñado.

\section{Conclusiones}

Tal y como muestran nuestros datos y los estudios citados, existe una relación muy estrecha entre las destrezas lectoras y la aptitud musical. Este hecho justifica y respalda la incorporación de videoclips subtitulados al aula de LE, beneficiando el desarrollo de competencias lingüísticas, musicales y de aprendizaje. Aunque el objeto de este estudio no es comprobar el efecto del uso de videoclips en las destrezas lectoras, si no animar a introducir su uso en las aulas, otros estudios anteriores sí han demostrado los beneficios de los videoclips en las aulas de lenguas (Berk, 2008; Gertrudix-Barrio y Gertrudix-Barrio, 2010; Gertrudix-Barrio y Rivas-Rebaque, 2015; Mericka, 2018; Sánchez-Vizcaino y Fonseca-Mora, 2018; Toscano-Fuentes y Julián de Vega, 2018).

Durante todo momento, el alumnado ha mostrado su deseo de colaborar en el estudio, por considerarlo útil y atractivo para su propio aprendizaje. La imagen, la voz, los subtítulos son tres pilares fundamentales para el aprendizaje de idiomas porque desarrollan la capacidad auditiva, pronunciación, ritmo, melodías, entonación al cantar; la lectura y la automatización de las correspondencias grafema-fonema a través de los subtítulos; y la comprensión gracias al apoyo de las imágenes. Por tanto, a través de este recurso multimodal se ha podido trabajar, a lo largo del proyecto, la comprensión auditiva y lectora, las relaciones letra-sonido, la pronunciación, la fluidez lectora; los elementos culturales y los mensajes que aparecían implícitos en las canciones.

Por otro lado, el hecho de que hayan tenido que seleccionar los videoclips que consumían y analizarlos desde el punto de vista cultural y didáctico les ha hecho reflexionar sobre lo importante y poderosa que son el análisis de las palabras y de las imágenes. Pues pueden llegar a transmitir valores negativos, homófobos, racistas, etc., contrarios a nuestro pensamiento pero que apoyamos, sin darnos cuenta, al cantar y bailar las canciones porque es lo comercial y lo que vende. Asimismo, la creación de kahoots ha beneficiado al alumnado en varios aspectos fundamentales: el aumento de input auditivo debido a las múltiples repeticiones para poder crear las diferentes actividades didácticas de comprensión; la 
posibilidad de crear una batería de preguntas de uso didáctico; también les ayudó a fomentar la competencia digital tan necesaria en la sociedad en la que vivimos.

Poder llevar a cabo experiencias similares en otros contextos y con más alumnado fortalecería los resultados que aquí se presentan. Sería, además, conveniente diseñar una intervención educativa adaptada al alumnado meta y medir las destrezas lectoras de los participantes tanto antes como después de la intervención educativa, para comprobar el auténtico alcance de dicha experiencia.

A pesar de las limitaciones de este estudio, consideramos que los resultados obtenidos son valiosos y abren muchas posibilidades pedagógicas. Por eso, en base a los resultados obtenidos, nos parece aconsejable que el profesorado introduzca los videoclips musicales en su práctica docente tanto en las aulas universitarias de lenguas extranjeras, como en niveles educativos anteriores. Es preciso que se introduzca la música y, sobre todo, el uso de los videoclips subtitulados en el aula de lenguas extranjeras, ya que son un recurso multimodal en el que se apela a los diferentes sentidos y llega a un gran número de estudiantes, pues la mayoría son consumidores activos de videoclips y de música en general. Además, trabajando con videoclips subtitulados se puede mejorar la fluidez lectora, ya que se automatiza la decodificación de palabras. Pero además, se accede al significado gracias al input visual, favoreciendo la comprensión en la LE, por lo que sería aconsejable introducirlos frecuentemente en el aula. Por otra parte, ayudaría a practicar elementos segmentales y suprasegmentales así como la pronunciación y lectura de los subtítulos, siempre que los tenga. Indudablemente se mejora la capacidad auditiva y lectora, puesto que recibe más input tanto oral como escrito. Por último, se fomenta el uso de las TICs y de las TACs, beneficiosas para su propio aprendizaje y para su futuro profesional.

Experiencias educativas similares a la presentada pueden ser útiles en cualquier etapa educativa, pues las pruebas pueden adaptarse según el nivel de competencia del estudiante y los videoclips pueden ser trabajados desde diferentes perspectivas, dependiendo del nivel lingüístico, el tipo de videoclips o el enfoque que quiera adoptar el docente, como dejar que los estudiantes seleccionen los videoclips o proponerlos él mismo. Es necesario subrayar el hecho de que los videoclips son un recurso motivador, que apela a las emociones, sobre todo, cuando es el propio alumnado el que selecciona con qué videoclip quiere trabajar. Sin embargo, durante la puesta en marcha de la experiencia educativa es imprescindible involucrar activamente al alumnado y proporcionarle pautas que le ayuden a reflexionar y a saber seleccionar sus propios recursos didácticos, para que desarrollen la competencia de aprender a aprender y mejoren su autonomía como aprendices de lenguas. En el caso de la formación de futuros docentes, estas experiencias también mejoran la capacidad crítica, la toma de decisiones y las habilidades para adaptar herramientas y recursos tecnológicos a las necesidades del alumnado meta. De modo que, al trabajar con ellas, las conviertan en recursos TAC que generen un aprendizaje de mayor calidad, lo cual reflejaría una formación docente que aspira a la excelencia. 


\section{Reconocimientos}

Este estudio ha sido financiado por el Ministerio de Economía y Competitividad de España a través del proyecto $\mathrm{I}+\mathrm{D}+\mathrm{i}$ «Aptitud musical, fluidez lectora y percepción intercultural de estudiantes universitarios europeos» Referencia: FFI2016-75452-R 1.

\section{Referencias}

Amitay, S., Ahissar, M. \& Nelken, I. (2002). Auditory processing deficits in reading disabled adults. Journal of the Association for Research in Otolaryngology, 3(3), 302-320.

Anvari, S. H., Trainor, L. J., Woodside, J., \& Levy, B. A. (2002). Relations among musical skills, phonological processing, and early reading ability in preschool children. Journal of Experimental Child Psychology, 83(2), 111-130. Recuperado de: https://bit.ly/2yJKS4K

Barwick, J., Valentine, E., West, R., \& Wilding, J. (1989). Relations between reading and musical abilities. British Journal of Educational Psychology, 59, $253-257$.

Berk, R. A. (2008). Star Tech: The Net Generation! Action in Teacher Education, 29 (56), 131-145. Recuperado de: https://bit.ly/2VFA5C1

Bolduc, J. (2008). The effects of music instruction on emergent literacy capacities among preschool children: A literature review. Early Childhood Research and Practice, 10 (1).

Bradley, L., \& Bryant, P. E. (1983). Categorizing sounds and learning to read - a causal connection. Nature, 301(5899), 419-421.

Cancelas-Ouviña, L.P. \& Herrera-Barba, J.M. (2014). Reflexiones sobre el potencial de YouTube en la enseñanza y aprendizaje de una Lengua Extranjera. Educación y Comunicación, 8, 71-82. Recuperado de: https://bit.ly/2yGoGsn

Council of Europe. (2001). Common European framework of reference for languages: Learning, teaching, assessment. Cambridge, U.K.: Press Syndicate of the University of Cambridge.

Chobert, J., \& Besson, M. (2013). Musical expertise and second language learning. Brain Sciences, 3(2), 923-940.

European values study EVS (1999). Spain, Field Questionnaire. Recuperado de: https://bit.ly/2KDOWWV 
Fernández-Corbacho, A. (2018). Desarrollo de destrezas lectoras en una lengua extranjera en educación primaria. Perfil del lector con dificultades. Tejuelo: Didáctica de la Lengua y la Literatura, 28, 91-126. Recuperado de: https://bit.ly/2XZGSYG

Foncubierta, J.M., Herrero Machancoses, F. \& Fonseca-Mora, M. C. (2018). La competencia lectora del alumnado universitario en contexto AICLE. Porta Linguarum, Monográfico III: Adressing bilingualism in Higher Education: policies and implementation issues, 75-88. Recuperado de: https://bit.ly/356ro6s

Fonseca-Mora, M. C. (2013). Melodías en el proceso de desarrollo de la capacidad lectora. Revista Electrónica De Estudios Filológicos, 25(2).

Fonseca-Mora, M.C. y Fernández-Corbacho, A. (2017). Procesamiento fonológico y aprendizaje de la lectura en lengua extranjera. Revista Española de Lingüística Aplicada/Spanish Journal of Applied Linguistics, 30(1), 166-187.

Foxton, J.M., Talcott, J.B., Witton, C., Brace, H., McIntyre, F., \& Griffiths, T.D. (2003). Reading skills are related to global, but not local, acoustic pattern perception. Nature Neuroscience, 6, 343-344

Gertrudix-Barrio, F. y Rivas Rebaque, B. (2015). Producción y diseño instructivo. Unaexperiencia de aprendizaje abierto y flipped classroom. Educatio Siglo XXI, 33 (1), 277-294. Recuperado de: https://bit.ly/3az6Ftf.

Gertrudix-Barrio, M. yGertrudix-Barrio, F. (2010). La utilidad de los formatos de interacciónmúsico-visual en la enseñanza. Comunicar, 17 (34), 99-107. Recuperado de: https://bit.ly/2VQ3VlO

Goswami, U. (1990). A special link between rhyming skill and the use of orthographic analogies by beginning readers. Journal of Child Psychology and Psychiatry, andAllied Disciplines, 31(2), 301-11. Recuperado de: https://bit.ly/2VClEyw

Goswami, U. (2005). Synthetic Phonics and Learning to Read: A Cross-language Perspective. Journal of Educational Psychology in Practice theory, research and practice ineducational psychology, 21(4).

Goswami, U. (2009). The Basic Processes. En D. R. Olson \& N. Torrance (Eds.), Reading: Insights from Neuroscience. The Cambridge Handbook of Literacy. Cambridge University Press.

Gough, P.B., \& Tunmer, W.E. (1986). Decoding, reading, and reading disability. Remedial and Special Education, 7(1), 6-10.

Hasbrouck, J., \& Tindal, G. A. (2006). Oral reading fluency norms: A valuable assessment tool for teaching teachers. The Reading Teacher, 59, 636-644 
Hoover, W.A., \& Gough, P.B. (1990). The simple view of reading. Reading and Writing, 2(2), 127-160

Jeon, E.H., \& Yamashita, J. (2014). L2 Reading comprehension and its correlates: A metaanalysis. Language Learning, 64(1), 160-212.

Kim, Y. S., Petscher, Y., \& Foorman, B. (2015). The unique relation of silent reading fluency to end-of-year reading comprehension: understanding individual differences at the student, classroom, school, and district levels. Reading and Writing, 28(1), 131-150

Kolb, A.Y. \& Kolb, D.A. (2008). Experiential Learning Theory: A Dynamic, Holistic Approach to Management Learning, Education and Development. En Armstrong, S. J. y Fukami, C. (Eds.) Handbook of Management Learning, Education and Development. London: Sage Publications 2008

Lessard, A. \& Hall, L. (2011). Links between Musical Learning and Reading for First to Third Grade students: A Literature Review Faculty of Education, University of Ottawa. Childhood a Global Journal of Child Research, 1 (7), 109-118.

Mericka, K. (2018). LipDub: a technology-enhanced language learning project with music. EnRosell-Aguilar, F., Beaven, T y Fuertes Gutiérrez, M. (Coord.), Innovative languageteaching and learning at university: integrating informal learning into formal language education,59-65. Recuperado de: https://bit.ly/352x7KC

Moreno, S., Marques, C., Santos, A., Santos, M., Castro, S. L., \& Besson, M. (2009). Musical training influences linguistic abilities in 8-year-old children: more evidence for brain plasticity. Cerebral Cortex (New York, N.Y.: 1991), 19(3), 712-723. Recuperado de: https://bit.ly/3aBghDO

National Institute of Child Health and Human Development (NICHD). (2000). Report of the National Reading Panel. Teaching children to read: An evidence-based assessment of the scientific research literature on reading and its implications for reading instruction: Reports of the subgroups (NIH Publication No. 00-4754). Washington, DC: U.S. Government Printing Office. Recuperado de: https://bit.ly/2Kx5nV5

Sánchez-Vizcaíno, M. C., \& Fonseca-Mora, M. C. (2019). Videoclip y emociones en el aprendizaje de Español como Lengua Extranjera. Círculo de Lingüística Aplicada a La Comunicación, 78, 255-286. Recuperado de: https://bit.ly/351TGiK

Scarborough, H. (2002). Connecting early language and literacy to later reading (dis)abilities: Evidence, theory and practice. En S. Neuman \& D. Dickinson (Eds.), Handbook of early literacy research, (pp. 97-110). London: The Guilford Press.

Segura, M. Candioti, C. \& Medina, J. (2007). Las TIC en la Educación. Panorama internacional y situación española CNICE-Fundación Santillana. Recuperado de 
http.//www.fundacionsantillana.org/Contenidos/Spain/SemanaMonografica/XXII/ DocumentoBasico.pdf

Sparks, R. L., Patton, J., Ganschow, L., Humbach, N., \& Javorsky, J. (2008). Early first language reading and spelling skills predict later second-language reading and spelling skills. Journal of Educational Psychology, 100(1), 162-174. Recuperado de: https://bit.ly/3bEzdmm

Standley, J.M. (2008). Does music instruction help children learn t oread? Evidence of a meta-analysis. Update: Applications of Research in Music Education, 27(1), 17- 32. Doi: $10.1177 / 8755123308322270$.

Tierney, A., Krizman, J. \& Kraus, N. (2015). Music Training alters the course of adolescent auditory development. National Academy of Sciences, 112(32). Doi: 10.1073/pnas.1505114112

Tierney, A., \& Kraus, N. (2013). Music training for the development of reading skills. In M. M. Merzenich, M. Nahum, T. M. Van Vleet (eds): Progress in Brain Research, Vol. 207, Burlington: Academic Press, 2013, pp. 209-241.

Toscano-Fuentes, C. M. (2010). Estudio empírico de la relación existente entre el nivel de adquisición de una segunda lengua, la capacidad auditiva y la inteligencia musical del alumnado. Tesis doctoral. Universidad de Huelva.

Toscano-Fuentes, C. \& Fonseca Mora, M.C. (2012). La música como herramienta facilitadora del aprendizaje del inglés como lengua extranjera. Teoría de la Educación, v.24, 2: 197-213.

Toscano-Fuentes, C.M. \& Julián-de-Vega, C. (2018). Vídeos musicales en el aula de inglés de primaria para la mejora de la fluidez lectora. Tejuelo, 28, 43-66.

Tucker, A. (1981). Music and the teaching of reading: A review of the literature. Reading Improvement, 18(1), 14.

Velasco Rodríguez, M.A. (2017). Las TAC y los recursos para generar aprendizaje. Infancia, Educación y Aprendizaje (IEYA), 3(2) (edición especial), 771-777. ISSN: 0719-6202. Recuperado de: https://bit.ly/2Y6fiZB

Ziegler, J.C. \& Goswami, U. (2006). Becoming literate in different languages: similar problems, different solutions. Developmental Science, 9(5), 429-436.

Amitay, S., Ahissar, M. \& Nelken, I. (2002). Auditory processing deficits in reading disabled adults. Journal of the Association for Research in Otolaryngology, 3(3), 302-320. 